\title{
MODELO DE LOCALIZAÇÃo HIERÁRQUICA DE USINAS DE PIRÓLISE
}

\author{
Ruan Carlos Alves Pereira \\ Universidade Federal Fluminense - UFF \\ ruancap@id.uff.br \\ Carlos Frederico Barros \\ Universidade Federal Fluminense - UFF \\ carlosfredericobarros@gmail.com \\ Anibal Alberto Vilcapoma Ignacio \\ Universidade Federal Fluminense - UFF \\ anibalvilcapoma@gmail.com
}

\begin{abstract}
Resumo
Este artigo visa identificar as localizações viáveis dentro de um grupo de municípios préselecionados para instalação de Usinas de pirólise Lenta a Tambor Rotativo, atividade considerada dinamizadora da economia e que tem por objetivo desenvolver um processo nacionalizado capaz de solucionar a irregular situação do tratamento de resíduos sólidos urbanos, solucionando os impactos negativos das disposições finais dos RSU em lixões etc. Para hierarquizar as localizações das usinas de pirólise, utilizaremos o modelo COPPECOSENZA de análise hierárquica. O modelo COPPE-COSENZA é basicamente uma operação com matrizes baseada no confronto da oferta territorial com a demanda industrial, para uma série de fatores de localização estudados. O modelo COPPE - COSENZA utiliza como base para estruturação de seus conceitos variáveis linguísticas aplicadas a logica Fuzzy.
\end{abstract}

Palavras chaves: Lógica Fuzzy; Modelo de Hierarquização; Usina de Pirólise; Solução para Resíduos Urbanos.

\begin{abstract}
This paper intend identify viable locations within a group of pre-selected cities to instalation of pyrolysis plants by slowly drum rotating, activity considered a stimulus to economy and have a objective to develop a nationalized process able to solver the irregular situation of municipal treatment solid waste, solving the negative impacts from final provisions of RSU in landfills and others. To prioritize pyrolysis plants locations, we use the COPPE-COSENZA model hierarchical Analysis. The COPPE-COSENZA model is basically as operation between the territorial supply and industrial demand, based in locations factors studied. The COPPECOSENZA model use as base linguistic variables applied to Fuzzy logic for struct their concepts.
\end{abstract}

Key words: Fuzzy Logic; Model Hierarchy; Pyrolysis plant; Solution to Municipal Waste. 


\section{INTRODUÇÃ̃O}

A localização industrial é uma preocupação tradicionalmente presente ao se iniciar um trabalho de instalação de um empreendimento, uma vez que a decisão das empresas ou projetos em estabelecerem-se em determinadas localidades tem impactos importantes para a organização da atividade produtiva. Nesse contexto, abre-se um debate importante sobre quais são as características das configurações produtivas, sociais e de infraestrutura que permitem que esses benefícios sejam mais bem aproveitados pelo empreendimento a ser instalado e pela sociedade.

Segundo Detro [5], os métodos de localização industrial têm como objetivo buscar a melhor localização para uma ou mais instalações que podem ser fábricas, centros de distribuição, lojas de varejo, entre outras. Para a análise de localização se faz a modelagem, formulação e solução de uma classe de problemas que pode ser descrita como situar instalações em determinado espaço (Revelle [9]).

Os problemas de localização têm como finalidade determinar a localização de instalações de modo que as "vantagens" máximas possam ser obtidas em virtude da própria localização, em termos de medidas de desempenho desejáveis.

Os problemas de localização podem ser abordados tanto ao nível de macrolocalização como ao nível de microlocalização. Ao nível de macrolocalização podemos mencionar as contribuições de Collins e Walker [2] (enfoque geográfico) e de Cosenza [1] e Luna [8] (enfoque econômico). No entanto existe uma gama de modelos normativos (enfoque microeconômico), que correspondem a problemas que podem ser formulados e resolvidos com base em técnicas de otimização discreta (Ignácio [6], Galvão [4]).

No presente artigo é usado como base o modelo de Cosenza [1], que analisa e traduz tanto as características exigidas pelo empreendimento como os benefícios ofertados pelas regiões pré-selecionadas, gerando um índice de conveniência para instalação do empreendimento para as regiões analisadas.

O presente trabalho visa identificar as localizações viáveis dentro de um grupo de municípios pré-selecionados para instalação de uma usina de pirólise lenta a tambor rotativo, atividade considerada dinamizadora da economia e que tem por objetivo desenvolver um processo nacionalizado capaz de solucionar a irregular situação do tratamento de resíduos sólidos urbanos, solucionando os impactos negativos da disposição em lixões e oferecendo uma forma sustentável para gerir os resíduos sólidos urbanos (RSU) de determinada localidade.

Eleita a macro região, o empreendimento deverá localizar-se nas proximidades do atual repositório, seja aterro, lixão, etc., por questões de logística, tendo em vista que parte dos insumos da usina de pirólise lenta a tambor rotativo serão provenientes do passivo ambiental já disponível no repositório. Para o desenvolvimento dos estudos, vamos utilizar como base o Modelo COPPE-COSENZA de localização industrial. O Modelo COPPECOSENZA utiliza para estruturação de seus conceitos variáveis linguísticas aplicadas à lógica fuzzy, que está vocacionada para a manipulação de conceitos mal definidos utilizando-se de variáveis linguísticas no lugar de variáveis numéricas.

\section{TECNOLOGIA DE PIRÓLISE LENTA A TAMBOR ROTATIVO}

Por definição, a pirólise consiste na degradação térmica de hidrocarbonetos na ausência de oxigênio. Este processo requer uma fonte externa de calor para aquecer a matéria, podendo fazer a temperatura variar de $300^{\circ} \mathrm{C}$ a mais de $1000^{\circ} \mathrm{C}$. Entretanto, tem se observado que qualquer processo térmico com temperaturas superiores a $300^{\circ} \mathrm{C}$ na ausência de oxigênio é considerado método de pirólise, o que torna o termo extremamente abrangente.

Inicialmente, é possível fazer uma distinção quanto aos parâmetros de operação, como tempo de residência dos resíduos e a temperatura a qual ele é submetido: 
- Pirólise Lenta - Processo a temperaturas superiores a $400^{\circ} \mathrm{C}$ e longos períodos de residência (40min - 1hora) em que a proporção dos produtos obtidos normalmente é $30 \%$ líquidos, $35 \%$ carbonáceos e $35 \%$ gases;

- Pirólise Rápida - Processo a temperatura entre $400^{\circ} \mathrm{C}$ e $600^{\circ} \mathrm{C}$ e períodos de residência curtos $(\mathrm{t}<2 \mathrm{seg}$.) em que a proporção dos produtos obtidos normalmente é de $75 \%$ líquidos, $12 \%$ carbonáceos e $13 \%$ gases; e

- Flash Pirólise - Processo a temperaturas superiores a $800^{\circ} \mathrm{C}$ e períodos de residência curtos ( $\mathrm{t} \sim 1$ seg.), a proporção dos produtos obtidos normalmente é de $5 \%$ líquidos, $10 \%$ carbonáceos e $85 \%$ gases.

A matéria orgânica presente no RSU é tratada termicamente por pirólise e convertida em gás de síntese (aproximadamente $90 \%$ da matéria orgânica seca) e coque/carvão (10\% restantes). Outros inorgânicos também estarão presentes nos rejeitos sólidos da pirólise. No total, os rejeitos sólidos da pirólise terão entre $10 \%$ e $15 \%$ do peso do resíduo bruto. Para funcionamento da usina é necessário à queima de cerca de $30 \%$ do gás de síntese gerado, fazendo com que este processo seja autossustentável. Os $70 \%$ de gás restante pode ser utilizado para geração de energia elétrica e térmica, ou então, diretamente em sistemas térmicos para gerar calor ou frio. Os rejeitos sólidos gerados pela usina de pirólise consistem, principalmente, de: (a) resíduos metálicos segregados na etapa de preparação do RSU para o reator de pirolise e (b) rejeitos inertes decorrentes da pirólise dos RSU no reator. Os rejeitos inertes decorrentes da pirólise dos RSU possuem expectativa de caracterização como "biochar". Essa denominação é atribuída ao composto inerte identificado na Bacia Amazônica em ilhas de solos ricos e férteis, chamados "terra preta", criados pelos indígenas. Para Maia, Madari e Novotny [10], o "biochar" pode também ser produzido através de pirólise ou gaseificação, processos em que a biomassa é submetida ao calor na ausência ou com a redução de oxigênio.

\section{MODELO FUZZY COPPE-COSENZA DE HIERARQUIZAÇÃO}

Elaborado com o objetivo de permitir estudos mais aprofundados na localização de empreendimentos, o Modelo COPPE-COSENZA é basicamente uma operação com matrizes baseada no confronto da oferta territorial com a demanda industrial para uma série de fatores de localização estudados. O modelo visa encontrar a melhor localização dentro das considerações segundo as alternativas pesquisadas. Partindo deste princípio, o nível de satisfação de um conjunto de fatores requeridos por um empreendimento é analisado considerando a disponibilidade desses fatores em diferentes territórios. Seu principal diferencial é possibilitar a análise de fatores não somente econômicos do projeto, reunindo variáveis quantitativas e qualitativas. O modelo é composto então por dois espaços fuzzy: um para o conjunto de demandas e outro para um conjunto de potencialidades regionais, ditas ofertas. Cada espaço definido por números fuzzy é delimitado por meio de graus de pertinência dos fatores para um determinado elemento do suporte. Os suportes para o requerimento dos fatores são estabelecidos em quatro níveis, a saber: Crítico, condicionante, pouco condicionante e irrelevante para a função de demanda $A$. Superior, bom, regular e fraco para a função de oferta $B$. Devido à ambiguidade, vagueza e imprecisão que caracterizam a demanda e a oferta (conjuntos fuzzy $A$ e $B$ ), as informações devem ser filtradas por experts e inferidas por meio de uma corrente cognitiva. A estrutura dos conjuntos $A$ e $B$, fatores de demanda e oferta são definidas de forma precisa pelas matrizes de relações de pertinência como se segue:

$$
A=(a i j)_{h x n} \text { e } B=(b j k)_{n x m}
$$


Nesta versão aij é um coeficiente fuzzy da demanda do projeto $i$ pelo fator de demanda $j$ e $b j k$ é um coeficiente fuzzy produzido por um atributo $j$ existente na localidade $k$. A matriz $A$, como sendo um conjunto fuzzy, pode ser representada por $\tilde{A}$, e a matriz $B$, representando números fuzzy, é representada por $\sim B$. As dimensões dessas matrizes supracitadas ainda representam respectivamente: $h$ diferentes tipos de empreendimentos por $n$ fatores de localização e a oferta destes fatores por $m$ alternativas de localização. A oferta de um fator em uma determinada região pode ser superior ou inferior ao nível demandado, onde, para cada caso há um valor de pertinência entre as variáveis.

Seja $\mathrm{F}=\{$ fi $\mid \mathrm{i}=1, \ldots, \mathrm{n}\}$ um conjunto finito de fatores de localização denominado genericamente de $f$. Então, o conjunto fuzzy $\tilde{A}$ em $\mathrm{F}$ é um conjunto de pares ordenado: $\tilde{A}=\left\{\left(f, \mu_{\tilde{A}}(f) \mid f \in F\right)\right\}$, onde $\tilde{A}$ é uma representação $f u z z y$ da matriz de demanda $A$, e $\mu_{\tilde{\AA}}(f)$ representa o grau de importância dos fatores, definidos entre crítico, condicionante, pouco condicionante ou irrelevante

Tabela 1 - Matriz A - Fatores demandados por tipo de empreendimento

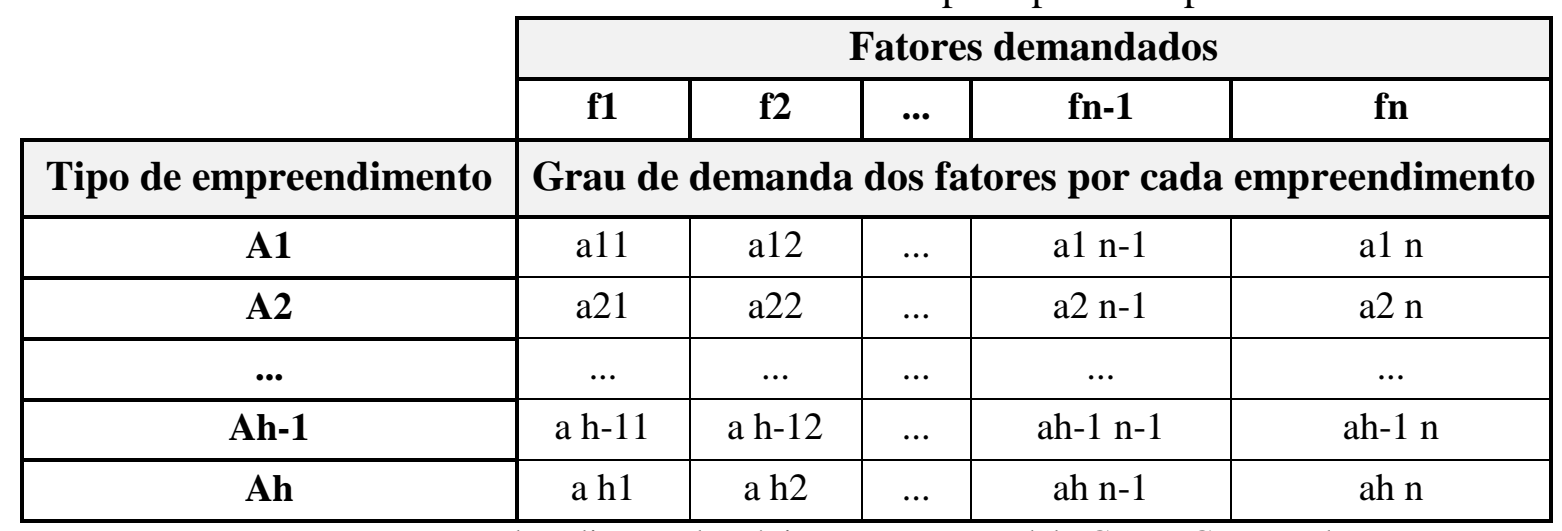

Fonte: Um caso de aplicação da Lógica Fuzzy- o Modelo Coppe-Cosenza de Hierarquia Fuzzy (2003)

Onde aij é um coeficiente fuzzy do projeto $h$ com relação ao fator $n$.

Similarmente, $\sim B=\left\{\left(f, \mu_{\sim B}(f) \mid f \in F\right)\right\}$, onde $\sim B$ é uma representação fuzzy da matriz de oferta $B$ e $\mu_{\sim B}(f)$ representa o grau de atendimento dos fatores disponíveis pelas diferentes alternativas de localização definidos entre superior, bom, regular ou fraco.

Tabela 2 - Matriz B - Fatores ofertados por cada localização

\begin{tabular}{|c|c|c|c|c|c|}
\cline { 2 - 6 } \multicolumn{1}{c|}{} & \multicolumn{5}{c|}{ Localizações } \\
\hline Fatores ofertados & B1 & B2 & $\ldots$ & Bm-1 & Bm \\
\hline f1 & b11 & b12 & $\ldots$ & b1m-1 & b1m \\
\hline f2 & b21 & b22 & $\ldots$ & b2m-1 & b2m \\
\hline$\ldots$ & $\ldots$ & $\ldots$ & $\ldots$ & $\ldots$ & $\ldots$ \\
\hline fn-1 & bn-11 & bn-12 & $\ldots$ & bn-1m-1 & bn-1m \\
\hline Fn & bn1 & bn2 & $\ldots$ & bnm-1 & bnm \\
\hline
\end{tabular}

Fonte: Um caso de aplicação da Lógica Fuzzy- o Modelo Coppe-Cosenza de Hierarquia Fuzzy (2003)

Onde bjk é um coeficiente fuzzy da localização $m$ em relação ao fator $n$.

Considerando dois elementos genéricos aij e $b j k$, o cotejo entre aij e bjk será obtido através do operador aij $\otimes b j k$. 
Tabela 3 - aij $\otimes b j k$ - Cotejo entre os fatores gerais de demanda e oferta

\begin{tabular}{|c|c|c|c|c|}
\hline aij $\boldsymbol{\otimes b j} \boldsymbol{k}$ & \multicolumn{4}{|c|}{ Oferta } \\
\hline Demanda & $\mathbf{A}$ & $\mathbf{B}$ & $\mathbf{C}$ & $\mathbf{D}$ \\
\hline $\mathbf{A}$ & 1 & $1-1 / \mathrm{n}$ & $1-2 / \mathrm{n}$ & 0 \\
\hline B & $1+1 / \mathrm{n}$ & 1 & $1-1 / \mathrm{n}$ & $1-2 / \mathrm{n}$ \\
\hline $\mathbf{C}$ & $1+2 / \mathrm{n}$ & $1+1 / \mathrm{n}$ & 1 & $1-1 / \mathrm{n}$ \\
\hline $\mathbf{D}$ & $1+3 / \mathrm{n}$ & $1+2 / \mathrm{n}$ & $1+1 / \mathrm{n}$ & 1 \\
\hline
\end{tabular}

Fonte: Um caso de aplicação da Lógica Fuzzy- o Modelo Coppe-Cosenza de

Hierarquia Fuzzy (2003)

Seja $C=\left(C_{i k}\right)_{h x m}=A_{h x n} \otimes B_{n x m}$, a matriz de possibilidades que representa a soma dos índices de localização do projeto $i$ nas $k$ zonas selecionadas. Assim, caso a aplicação do Modelo contar apenas com fatores gerais e não depender de fatores específicos para sua realização, $\operatorname{Maxi}\{C i k\}=C i$, indica a melhor localização do projeto $i$, no conjunto de ofertas territoriais e $\operatorname{Maxk}\{C i k\}=C k$, indica o melhor tipo de projeto para as $k$ zonas selecionadas.

Nas diferentes aplicações, o modelo pode ser generalizado em vários sentidos sem perder suas características básicas. Em situações onde o empreendimento exija características específicas para sua instalação, deverão ser utilizados fatores de demanda específicos $A^{*}$, que irão compor a matriz $C^{*}$. Os fatores específicos são fatores essenciais para a vitalidade do empreendimento ou projeto, sendo estritamente restritivos. A matriz de possibilidades segundo critérios específicos $C^{*}{ }_{h x m}$ é resultante do confronto entre a matriz de demanda e a matriz de oferta de critérios específicos, onde: $C^{*}=\left(C_{i k}\right)_{h x m}=A^{*}{ }_{h x r} \otimes B^{*}{ }_{r x m}$. matriz $A^{*}$.

Seja $A^{*}=\left(a^{*} i j\right)_{h x r}$, onde, $\tilde{A}^{*}=\left\{\left(f, \mu_{\tilde{\AA}^{*}}(f) \mid f \in F\right)\right\}$, é a representação fuzzy da

Assumindo $B^{*}=\left(b^{*} j k\right)_{r x m}$ a matriz de fatores de oferta territorial de fatores específicos. Onde, $\sim B^{*}=\left\{\left(f, \mu_{\sim B^{*}}(f) \mid f \in F\right)\right\}$, é a representação fuzzy da matriz $B^{*}$.

$\mathrm{O}$ cotejo entre demanda e oferta de fatores específicos segue a mesma lógica que os fatores gerais.

Tabela $4-a * i j \otimes b * j k$ - Cotejo entre os fatores específicos de demanda e oferta.

\begin{tabular}{|c|c|c|c|c|}
\hline $\boldsymbol{a} * \boldsymbol{i} \boldsymbol{j} \otimes \boldsymbol{b} * \boldsymbol{j} \boldsymbol{k}$ & \multicolumn{5}{|c|}{ Oferta } \\
\hline Demanda & $\mathbf{A}$ & $\mathbf{B}$ & $\mathbf{C}$ & $\mathbf{D}$ \\
\hline $\mathbf{A}$ & 1 & $1-1 / \mathrm{n}$ & $1-2 / \mathrm{n}$ & 0 \\
\hline $\mathbf{B}$ & $1+1 / \mathrm{n}$ & 1 & $1-1 / \mathrm{n}$ & $1-2 / \mathrm{n}$ \\
\hline $\mathbf{C}$ & $1+2 / \mathrm{n}$ & $1+1 / \mathrm{n}$ & 1 & $1-1 / \mathrm{n}$ \\
\hline $\mathbf{D}$ & $1+3 / \mathrm{n}$ & $1+2 / \mathrm{n}$ & $1+1 / \mathrm{n}$ & 1 \\
\hline
\end{tabular}

Fonte: Um caso de aplicação da Lógica Fuzzy- o Modelo Coppe-Cosenza de

Hierarquia Fuzzy (2003)

É importante ressaltar que a provável não satisfação de algum critério específico impossibilita a seleção da alternativa da categoria demandante.

A matriz de agregação de possibilidades, $\Gamma_{h x m}$, (Tabela 5) reúne as duas matrizes de possibilidades descritas anteriormente, $C$ e $C^{*}$, apresentando as situações de abundância ou carência em relação à viabilidade das alternativas consideradas. Esta matriz $\Gamma_{h x m}$ é resultante da seguinte operação: $\Gamma=(\gamma i k)_{h x m}=C_{h x m}+C^{*}{ }_{h x m}$, onde cada $\gamma i k$ resulta da operação a seguir. 
Tabela $5-\Gamma_{h x m}-$ Matriz de agregação de possibilidades.

\begin{tabular}{|c|c|c|}
\hline$c i k$ & $>0$ & 0 \\
\hline$c^{*} i k$ & 0 & 0 \\
\hline 0 & $c i k+c{ }^{\star} i k$ & $c{ }^{*} i k$ \\
\hline
\end{tabular}

Fonte: Localização de curtumes no Brasil através do modelo COPPE-COSENZA de localização industrial Marco Cristellotti - 2011

A matriz $\Gamma_{h x m}$ descrita acima fornece informações a respeito da eficiência das alternativas consideradas, permitindo assim a devida escolha das melhores alternativas segundo os valores acumulados por $C$ e $C^{*}$, de modo que:

$\gamma \mathrm{i}=\operatorname{Maxi}\{\gamma \mathrm{ik}\}$ indica a melhor alternativa locacional $k$, para cada empreendimento $i$; $\gamma \mathrm{k}=\operatorname{Maxk}\{\gamma \mathrm{ik}\}$ indica o melhor empreendimento $i$, para cada alternativa locacional $k$.

Assim, é possível indicar o empreendimento que melhor se adeque em cada uma das regiões pré-selecionadas e determinar a região mais adequada para cada tipo de empreendimento.

\section{CASO APLICADO - LOCALIZAÇÃO DE USINAS DE PIRÓLISE}

A região alvo do presente estudo são cidades localizadas no sul de Minas gerais, no entorno do lago de Furnas. O projeto de pesquisa e desenvolvimento (P\&D) onde foi realizada a aplicação do modelo ressalta que as usinas de pirólise deverão ser instaladas em determinadas localidades onde atendam aos 52 municípios, pois o P\&D tem como objetivo o desenvolvimento de unidades de aproveitamento energético de resíduos através de tecnologia de pirólise lenta a tambor rotativo, na aplicação de solução socioambiental. Os municípios analisados serão nomeados como "Cidade n", onde n variará de 1 a 52. Após a aplicação do modelo, cada município será representado por um índice que refletirá sua necessidade e capacidade para receber uma usina. Para compor a matriz $C$, serão utilizados fatores que refletem a necessidade de uma localidade em receber uma usina. Já a composição da matriz $C^{*}$ refere-se a fatores relacionados ao funcionamento da usina.

Para representar $\Gamma_{h x m}$ onde originalmente $\Gamma=(j i k)_{h x m}=C_{h x m}+C^{*}{ }_{h x m}$, será utilizada a equação onde $\Gamma=(j i k)_{h x m}=C_{h x m}-C^{*}{ }_{h x m}$.

A alteração na equação original se fez necessária, pois há uma diferença entre os aspectos refletidos pela matriz $C$ e pela matriz $C^{*}$. A matriz $C$ mostra o quão eficiente é determinada cidade em relação aos fatores demandados. Por exemplo, a destinação final de RSU de uma cidade é um fator de alta importância (crítico) e quanto menos estruturada for a destinação final de RSU na cidade, menor será a contribuição para o somatório gerador da matriz $C$. Logo, quanto mais deficitária a cidade, mais urgentemente deverão ser solucionados seus problemas socioambientais, contribuindo para a priorização em recebimento de uma usina de pirolise frente às outras localidades. Por outro lado, a matriz $C^{*}$ informa em que níveis uma cidade apresenta a relação de oferta e demanda de fatores necessários ao funcionamento de uma usina, sendo necessário atingir um nível mínimo de oferta desses fatores para o bom funcionamento da mesma. Para que a usina apresente um bom funcionamento é necessário, por exemplo, que a região ofereça um nível mínimo de coleta de RSU na cidade, visto que os resíduos precisam chegar até a usina de pirólise para serem tratados. Assim, caso a coleta de RSU for bem estruturada na cidade, irá contribuir para tal 
cidade receber uma usina de pirólise, contudo, se esta cidade ultrapassa o nível mínimo requerido desse fator, passa a ser, menos necessitada de uma usina de pirolise como solução socioambiental, frente às outras cidades. Deste modo, para representar $\Gamma_{h x m}$ utiliza-se, neste caso, $\Gamma=(\gamma i k)_{h x m}=C_{h x m}-C_{h x m}^{*}$, gerando um índice de prioridade para recebimento de uma usina de pirólise. Quanto menor for o valor de $\Gamma_{h x m}$ para uma cidade, antes dever-se-á receber uma usina tal cidade.

\subsection{MENSURAÇÕES DE OFERTA E DEMANDA DE FATORES}

Para cada fator de localização considerado no estudo, é elaborada uma ficha contendo sua conceituação e os parâmetros pelos quais devem ser mensurados, tanto na mensuração da oferta como na demanda.

\subsection{COMPOSIÇÕES DA MATRIZ $C$}

Os fatores que irão compor a matriz $C$ serão analisados conforme sua oferta e demanda para cada região pré-selecionada. Tais fatores são demandados em determinados níveis, como será mostrado adiante, e quanto menos a cidade atende a esses fatores demandados, piores são seus índices fuzzy gerados, e mais necessitada de uma usina torna-se a cidade. Esses fatores foram eleitos através de experiência dos responsáveis pela instalação da planta de usina de pirólise.

\subsubsection{FATORES DE INFLUÊNCIA PARA MATRIZ $C$}

- Disposição final RSU;

- Disposição final rural;

- Disposição de RSS;

- Tempo de vida do repositório;

- Presença de Área contaminada (lixão desativado);

- Distancia de corpos d'água ou fontes de água;

- Profundidade de lençóis freáticos e aquíferos;

- Localização sob aspecto de área ocupada (Informa se há moradias, escolas, hospitais ou outros tipos de áreas humanamente ocupadas próximo ao local de destinação final dos resíduos);

- Proximidade a unidades de conservação;
- Volume de RSS;

- Volume de lixo agrossilvopastoril;

- Nível da coleta seletiva;

- Existência de cooperativa ou associação de catadores;

- Presença de unidades de destinação especifica;

- Educação ambiental;

- População flutuante devido ao Interesse turístico;

- Taxa de crescimento populacional.

Com base nos fatores gerais listados é possível estruturar a matriz $A$ e $B$. Em seguida realizar o cotejo entre elas gerando a matriz $C$.

Tabela 6 - Fatores gerais

\begin{tabular}{|l|l|}
\hline \multicolumn{2}{|c|}{ Fatores Gerais } \\
\hline F1 & Disposição final RSU \\
\cline { 2 - 3 } F2 & Disposição final rural \\
\cline { 2 - 2 } F3 & Disposição de RSS \\
\cline { 2 - 2 } F4 & Tempo de vida do repositório \\
\cline { 2 - 2 } F5 & Presença de área contaminada \\
\cline { 2 - 2 } F6 & Distancia de corpos d'água ou fontes de água \\
\cline { 2 - 3 } F7 & Profundidade de lençóis freáticos e aquíferos \\
\cline { 2 - 2 } &
\end{tabular}




\begin{tabular}{|l|l|} 
F8 & Localização sob aspecto de área ocupada \\
\cline { 2 - 3 } F9 & Proximidade a unidades de conservação \\
\cline { 2 - 3 } F10 & Volume de RSS \\
\cline { 2 - 3 } F11 & Volume de lixo agrossilvopastoril \\
\cline { 2 - 2 } F12 & Nível da coleta seletiva \\
\cline { 2 - 2 } F13 & Existência de cooperativa, associação de catadores \\
F14 & Presença de unidades de destinação especifica \\
\cline { 2 - 2 } F15 & Educação ambiental \\
\cline { 2 - 2 } F16 & População flutuante devido ao turismo \\
\cline { 2 - 2 } F17 & Taxa de crescimento populacional \\
\hline
\end{tabular}

Fonte: Própria (2014)

Tabela 7 - Matriz A utilizada na aplicação

\begin{tabular}{|l|c|}
\hline \multicolumn{2}{|c|}{ Empreendimento - Usina de Pirólise } \\
\hline Fisposição final RSU & Importância associada aos fatores \\
\hline Disposição final rural & Critico \\
\hline Disposição de RSS & Condicionante \\
\hline Tempo de vida do repositório & Pouco condicionante \\
\hline Presença de área contaminada & Critico \\
\hline Distancia de corpos d'água ou fontes de água & Critico \\
\hline Profundidade de lençóis freáticos e aquíferos & Critico \\
\hline Localização sob aspecto de área ocupada & Critico \\
\hline Proximidade a unidades de conservação & Pritico \\
\hline Volume de RSS & Pouco condicionante \\
\hline Volume de lixo agrossilvopastoril & Critico \\
\hline Nível da coleta seletiva & Pouco condicionante \\
\hline Existência de coop. ou assoc. de catadores & Pouco condicionante \\
\hline Presença de unid. de destinação especificas & Pouco condicionante \\
\hline Educação ambiental & Pouco condicionante \\
\hline População flutuante devido ao turismo & Pouco condicionante \\
\hline Taxa de crescimento populacional & \\
\hline
\end{tabular}

Fonte: Própria (2014)

Tabela 8 - Matriz $B$ utilizada na aplicação

\begin{tabular}{|c|c|c|c|c|c|c|c|}
\hline $\begin{array}{c}\text { Fatores Gerais } \\
\text { Oferecidos }\end{array}$ & Cidade 1 & Cidade 2 & Cidade 3 & $\ldots$ & Cidade 50 & Cidade 51 & Cidade 52 \\
\hline F1 & bom & regular & regular & $\ldots$ & regular & bom & bom \\
\hline F2 & regular & regular & bom & $\ldots$ & regular & regular & bom \\
\hline F3 & fraco & fraco & superior & $\ldots$ & bom & fraco & regular \\
\hline F4 & bom & bom & fraco & $\ldots$ & bom & bom & regular \\
\hline F5 & fraco & fraco & bom & $\ldots$ & bom & fraco & superior \\
\hline
\end{tabular}




\begin{tabular}{|c|c|c|c|c|c|c|c|} 
F6 & bom & bom & superior & $\ldots$ & bom & bom & superior \\
\hline F7 & fraco & fraco & bom & $\ldots$ & bom & fraco & superior \\
\hline F8 & fraco & fraco & fraco & $\ldots$ & bom & fraco & superior \\
\hline F9 & regular & bom & fraco & $\ldots$ & bom & regular & bom \\
\hline F10 & regular & bom & bom & $\ldots$ & bom & regular & bom \\
\hline F11 & bom & superior & superior & $\ldots$ & bom & bom & bom \\
\hline F12 & bom & fraco & fraco & $\ldots$ & regular & bom & bom \\
\hline F13 & bom & bom & bom & $\ldots$ & fraco & bom & bom \\
\hline F14 & bom & superior & superior & $\ldots$ & fraco & bom & bom \\
\hline F15 & regular & bom & bom & $\ldots$ & bom & regular & regular \\
\hline F16 & bom & fraco & bom & $\ldots$ & superior & superior & bom \\
\hline F17 & bom & bom & bom & $\ldots$ & fraco & fraco & regular \\
\hline
\end{tabular}

Fonte: Própria (2014)

O cotejo entre as matrizes $A$ e $B$ são feitos para cada município. Abaixo segue o exemplo para a cidade 1 .

Tabela $9-A_{h x n} \otimes \mathrm{B}_{n x m}-$ Cidade 1

\begin{tabular}{|l|c|c|c|}
\hline \multicolumn{1}{|c|}{$\boldsymbol{A}_{\boldsymbol{h x \boldsymbol { n }}} \otimes \mathbf{B}_{\boldsymbol{n} \boldsymbol{x \boldsymbol { m }}}$} & Demanda & Oferta & $\begin{array}{c}\text { Índice fuzzy por fator } \\
\text { de demanda }\end{array}$ \\
\hline Disposição final RSU & Critico & bom & $\mathbf{0 , 9 4}$ \\
\hline Disposição final rural & Condicionante & regular & $\mathbf{0 , 9 4}$ \\
\hline Disposição de RSS & Condicionante & fraco & $\mathbf{0 , 8 8}$ \\
\hline Tempo de vida do repositório & Pouco condicionante & bom & $\mathbf{1 , 0 6}$ \\
\hline Presença de área contaminada & Critico & fraco & $\mathbf{0 , 0 0}$ \\
\hline Distancia de corpos d’água ou fontes de água & Critico & bom & $\mathbf{0 , 9 4}$ \\
\hline Profundidade de lençóis freáticos e aquíferos & Critico & fraco & $\mathbf{0 , 0 0}$ \\
\hline Localização sob aspecto de área ocupada & Critico & fraco & $\mathbf{0 , 0 0}$ \\
\hline Proximidade a unidades de conservação & Critico & regular & $\mathbf{0 , 8 8}$ \\
\hline Volume de RSS & Pouco condicionante & regular & $\mathbf{1 , 0 0}$ \\
\hline Volume de lixo agrossilvopastoril & Pouco condicionante & bom & $\mathbf{1 , 0 6}$ \\
\hline Nível da coleta seletiva & Critico & bom & $\mathbf{0 , 9 4}$ \\
\hline Existência de coop. ou assoc. de catadores & Pouco condicionante & bom & $\mathbf{1 , 0 6}$ \\
\hline Presença de unid. de destinação especificas & Pouco condicionante & bom & $\mathbf{1 , 0 6}$ \\
\hline Educação ambiental & Pouco condicionante & regular & $\mathbf{1 , 0 0}$ \\
\hline População flutuante devido ao turismo & Pouco condicionante & bom & $\mathbf{1 , 0 6}$ \\
\hline Taxa de crescimento populacional & Pouco condicionante & bom & $\mathbf{1 , 0 6}$ \\
\hline
\end{tabular}

Fonte: Própria (2014)

A matriz $C$ (Tabela 10) gera para cada localidade um índice que é calculado através do somatório dos índices fuzzy gerados no cotejo entre a demanda e a oferta dos fatores gerais.

Tabela 10 - Matriz $C$

\begin{tabular}{|c|c|}
\hline \multicolumn{2}{|c|}{ Matriz C } \\
\hline Cidade 1 & 13,88 \\
\hline
\end{tabular}




\begin{tabular}{|c|c|}
\cline { 1 - 1 } Cidade 2 & 13,06 \\
\cline { 1 - 1 } Cidade 3 & 14,29 \\
\cline { 1 - 1 }$\ldots$ & $\ldots$ \\
\cline { 1 - 1 } Cidade 50 & 16,59 \\
\cline { 1 - 1 } Cidade 51 & 13,82 \\
\cline { 1 - 1 } Cidade 52 & 17,06 \\
\cline { 1 - 1 } Fonte: Própria (2014)
\end{tabular}

\subsection{COMPOSIÇÕES DA MATRIZ $C^{*}$}

Os fatores selecionados que irão compor a matriz $C^{*}$ são demandados em níveis mínimos, tendo caráter restritivo caso não for atingido e caráter penalizador para quando ultrapassa o nível requerido. Esses foram eleitos através de experiência dos integrantes do $\mathrm{P} \& \mathrm{D}$, responsáveis pela instalação da planta piloto de usina de pirólise.

\subsubsection{FATORES DE INFLUÊNCIA PARA MATRIZ $C$ *}

- Volume de passivo ambiental (atual local de destinação final dos RSU);

- Volume de produção de lixo;

- Coleta do RSU.

Já descritos os fatores específicos, é possível estruturar as matrizes $A^{*}, B^{*}$, realizar o cotejo entre elas e gerar a matriz $C^{*}$.

Tabela 11 - Fatores específicos

\begin{tabular}{|c|c|}
\hline \multicolumn{2}{|c|}{ Fatores Específicos } \\
\hline F1' & Volume de passivo (atual lixão) \\
\cline { 2 - 2 } F2' $^{\prime}$ & Volume de produção de lixo \\
\cline { 2 - 2 } F3' $^{\prime}$ & Coleta de RSU \\
\hline
\end{tabular}

Tabela 12 - Matriz $A^{*}$ utilizada na aplicação

\begin{tabular}{|c|c|}
\hline \multicolumn{2}{|c|}{ Empreendimento - Usina de Pirólise } \\
\hline Fatores específicos demandados & Importância associada aos fatores \\
\hline Volume de passivo (atual lixão) & Irrelevante \\
\hline Volume de produção de lixo & Pouco condicionante \\
\hline Coleta de RSU & Condicionante \\
\hline
\end{tabular}

Fonte: Própria (2014)

Tabela 13 - Matriz B* utilizada na aplicação

\begin{tabular}{|c|c|c|c|c|c|c|c|}
\hline $\begin{array}{c}\text { Fatores Específicos } \\
\text { Oferecidos }\end{array}$ & Cidade 1 & Cidade 2 & Cidade 3 & .. & Cidade 50 & Cidade 51 & Cidade 52 \\
\hline F1' & superior & bom & bom & $\ldots$ & bom & fraco & bom \\
\hline F2' & regular & regular & superior & $\ldots$ & regular & regular & regular \\
\hline F3' & bom & bom & bom & $\ldots$ & regular & bom & bom \\
\hline
\end{tabular}


$\mathrm{O}$ cotejo entre as matrizes $\mathrm{A}^{*}$ e $\mathrm{B}^{*}$ serão realizados para cada município. Segue abaixo o exemplo para cidade 1 .

Tabela $14-A^{*}{ }_{h x n} \otimes \mathrm{B}^{*}{ }_{n x m}-$ Cidade 1

\begin{tabular}{|c|c|c|c|}
\hline$A^{*}{ }_{h x n} \otimes \mathrm{B}^{*}{ }_{n x m}$ & Demanda & Oferta & Índice Fuzzy por fator de demanda \\
\hline Volume de passivo (atual / ativo) & Irrelevante & superior & $\mathbf{2 , 0}$ \\
\hline Volume de produção de lixo & Pouco condicionante & regular & $\mathbf{1 , 0}$ \\
\hline Nível de Coleta do lixo & Condicionante & bom & $\mathbf{1 , 0}$ \\
\hline
\end{tabular}

Fonte: Própria (2014)

A Matriz $C^{*}$ gera para cada localidade um índice que é calculado através do somatório dos índices Fuzzy gerados no cotejo entre a demanda e a oferta dos fatores específicos.

Tabela 15 - Matriz $C^{*}$

\begin{tabular}{|c|c|}
\hline \multicolumn{2}{|c|}{ Matriz $\boldsymbol{C}^{*}$} \\
\cline { 1 - 1 } Cidade 1 & 4,00 \\
\cline { 1 - 1 } Cidade 2 & 3,67 \\
\cline { 1 - 1 } Cidade 3 & 4,33 \\
\cline { 1 - 1 }$\ldots$ & $\ldots$ \\
\cline { 1 - 1 } Cidade 50 & 3,67 \\
\cline { 1 - 1 } Cidade 51 & 3,00 \\
\cline { 1 - 1 } Cidade 52 & 3,67 \\
\hline \multicolumn{2}{|c|}{ Fonte: Própria (2014) }
\end{tabular}

Para expressar o resultado utilizaremos a matriz $\Gamma_{h x m}$ que consiste na análise entre as matrizes $C$ e $C^{*}$.

Tabela 16 - Matriz $\Gamma_{h x m}$

\begin{tabular}{|c|c|c|c|c|c|c|c|}
\cline { 2 - 8 } \multicolumn{1}{c|}{} & Cidade 1 & Cidade 2 & Cidade 3 & $\ldots$ & Cidade 50 & Cidade 51 & Cidade 52 \\
\hline Matriz C & 13,88 & 13,06 & 14,29 & & 16,59 & 13,82 & 17,06 \\
\hline Matriz C* & 4,00 & 3,67 & 4,33 & $\ldots$ & 3,67 & 3,00 & 3,67 \\
\hline Matriz $\boldsymbol{\Gamma}$ & 9,88 & 9,39 & 9,96 & $\ldots$ & 12,92 & 10,82 & 13,39 \\
\hline
\end{tabular}

Fonte: Própria (2014)

A partir da matriz $\Gamma_{h x m}$ é possível listar em forma de ranking as localidades a receberem as usinas. Neste caso, não houveram localidades restringidas, mas caso houvesse, estariam relacionadas ao não atendimento a algum fator específico de forma mínima.

Como descrito anteriormente, quanto menor for o valor de $\Gamma_{h x m}$ para uma cidade, antes dever-se-á receber uma usina tal cidade. Segue abaixo o ranking de prioridade de instalação das usinas de pirólise:

Tabela 17 - Ranking

\begin{tabular}{|c|c|}
\hline Localidades & Prioridade \\
\hline Cidade 2 & $1^{\text {o }}$ \\
\hline Cidade 1 & $2^{\text {o }}$ \\
\hline Cidade 3 & $3^{\text {o }}$ \\
\hline$\ldots$ & $\ldots^{\text {o }}$ \\
\hline Cidade 51 & $4^{\text {o }}$ \\
\hline Cidade 50 & $5^{\circ}$ \\
\hline Cidade 52 & $6^{\circ}$ \\
\hline
\end{tabular}

Fonte: Própria (2014) 


\section{CONCLUSÃO}

É relevante afirmar que o principal diferencial do Modelo é a incorporação de variáveis externas à análise puramente econômica do empreendimento em questão. Sendo, portanto, uma ferramenta de apoio à decisão única capaz de reunir variáveis qualitativas e quantitativas, facilitando seu uso, podendo ser adequado às especificidades de cada projeto.

Portanto, abre-se um infindável leque de aplicações, onde a maior preocupação do analista de projeto deve ser a formatação do sistema, de modo a garantir a consistência e confiabilidade dos resultados.

Vale a pena ressaltar que o modelo aqui aplicado não indica necessariamente a melhor alternativa, e sim possibilidades de localização. Ou seja, indica a melhor alternativa em termos de oferta de fatores locacionais. É importante lembrar que o P\&D no qual foi utilizado o modelo tem o objetivo de desenvolver unidades de aproveitamento energético através tecnologia de pirólise lenta à tambor rotativo na aplicação de solução socioambiental, por isso muitos fatores levam em conta a possibilidade de desenvolvimento e aspectos que possam maximizar os ganhos sociais da região. Assim, em alguns casos, é possível verificar que uma região que não seria a mais indicada para instalação do empreendimento, acaba sendo priorizada, seja por conta de incentivos fiscais, ou outras demandas. Concluindo, queremos enfatizar que as informações produzidas pelo modelo utilizado neste estudo ajudam em muito, tanto aos planejadores governamentais, quanto aos empresários nas suas decisões.

\section{REFERÊNCIAS BIBLIOGRÁFICAS}

[1] COSENZA, C.A.N. et al. Localização Industrial: Delineamento de uma Metodologia para a Hierarquização das Potencialidades Regionais. Rio de Janeiro: COPPE/UFRJ. 1998

[2] COLLINS, L. e WALKER, D.F., (1975). Locational Dynamics of Manufacturing Activity, John Wiley \& Sons.

[3] CRISTELlOTTI, Marcos. Localização de curtumes no Brasil através do modelo CoppeCosenza de localização industrial. Dissertação (Mestrado) - Programa de Pós-graduação em Engenharia de Produção COPPE, da UFRJ, Rio de Janeiro, 2011.

[4] GALVÃO, R.D., ESPEJO, L.G.A., BOFFEY, B., 2002, "A hierarchical model for the location of perinatal facilities in the municipality of Rio de Janeiro", European Hournal of Operational Research, v. 138, pp. 495-517.

[5] DETRO, S. P. Otimização na Localização e na Capacidade de armazenamento de soja e milho para o estado do Paraná. PUC-PR. Curitiba, 2013.

[6] IGNACIO, A. A. V. ; FERREIRA FILHO, V. J. M. ; Sampaio, L.M.D . Modelos de localização hierárquicos na localização de plataformas de produção na indústria de petroleo e gas. In: Simpósio de Engenharia de Produção, 2012, Bauru -SP. XIX SIMPEP, 2012

[7] MARTINS, Guilherme Weber. Uma Contribuição aos estudos de localização industrial: Determinando o potencial de transporte aéreo de uma região com base no modelo de análise hierárquica Coppe-Cosenza. Dissertação (Mestrado) - Programa de Pós-graduação em Engenharia de Produção COPPE, da UFRJ, Rio de Janeiro, 2010.

[8] LUNA, H.P.L., (1979). Alguns Modelos Espaciais de Interesse para o Setor Agrícola, Monografia no. 1/79, Depto. de Ciências da Computação, UFMG

[9] REVELLE, C. S.; EISELT, H. A. Location analysis: A synthesis and survey. European Journal of Operational Research. Vol. 165, 1-19, 2005

[10] MAIA, C.M.B.F; MADARI, B.E. \& NOVOTNY, E. Advances in Biochar Research in Brazil. Dynamic Soil, Dynamic Plant. 5 (Special Issue 1), 53-58, 2011

[11] TOLEDO, O.M.; COSENZA, C.A.N . Um caso de aplicação da Lógica Fuzzy - o Modelo Coppe-Cosenza de Hierarquia Fuzzy. In: Encontro nacional de engenharia de produção, 2003, Ouro preto- MG. ENEGEP, 2003 